Long-term optical flux and colour variability in quasars

This content has been downloaded from IOPscience. Please scroll down to see the full text. 2016 Res. Astron. Astrophys. 16007

(http://iopscience.iop.org/1674-4527/16/2/007)

View the table of contents for this issue, or go to the journal homepage for more

Download details:

IP Address: 14.139.155.11

This content was downloaded on 09/08/2016 at 10:21

Please note that terms and conditions apply. 


\title{
Long-term optical flux and colour variability in quasars
}

\author{
N. Sukanya ${ }^{1}$, C. S. Stalin ${ }^{2}$, S. Jeyakumar ${ }^{3}$, D. Praveen ${ }^{4}$, Arnab Dhani $^{5}$ and R. Damle ${ }^{1}$ \\ 1 Department of Physics, Bangalore University, Bangalore 560 056, India; sukanya.2182@ gmail.com \\ 2 Indian Institute of Astrophysics, Bangalore 560 034, India \\ 3 Departmento de Astronomia, Universidad de Guanajuato, Mexico \\ 4 Department of Physics, Amrita School of Engineering, Bangalore 560 035, India \\ 5 Indian Institute of Technology, Roorkee 247 667, India
}

Received 2015 May 15; accepted 2015 July 21

\begin{abstract}
We have used optical $V$ and $R$ band observations from the Massive Compact Halo Object (MACHO) project on a sample of 59 quasars behind the Magellanic clouds to study their long term optical flux and colour variations. These quasars, lying in the redshift range of $0.2<z<2.8$ and having apparent $V$ band magnitudes between 16.6 and $20.1 \mathrm{mag}$, have observations ranging from 49 to 1353 epochs spanning over $7.5 \mathrm{yr}$ with frequency of sampling between 2 to 10 days. All the quasars show variability during the observing period. The normalised excess variance $\left(F_{\text {var }}\right)$ in $V$ and $R$ bands are in the range $0.2 \%<$ $F_{\text {var }}^{V}<1.6 \%$ and $0.1 \%<F_{\text {var }}^{R}<1.5 \%$ respectively. In a large fraction of the sources, $F_{\text {var }}$ is larger in the $V$ band compared to the $R$ band. From the z-transformed discrete cross-correlation function analysis, we find that there is no lag between the $V$ and $R$ band variations. Adopting the Markov Chain Monte Carlo (MCMC) approach, and properly taking into account the correlation between the errors in colours and magnitudes, it is found that the majority of sources show a bluer when brighter trend, while a minor fraction of quasars show the opposite behaviour. This is similar to the results obtained from another two independent algorithms, namely the weighted linear least squares fit (FITEXY) and the bivariate correlated errors and intrinsic scatter regression (BCES). However, the ordinary least squares (OLS) fit, normally used in the colour variability studies of quasars, indicates that all the quasars studied here show a bluer when brighter trend. It is therefore very clear that the OLS algorithm cannot be used for the study of colour variability in quasars.
\end{abstract}

Key words: time lag — periodicity — active galaxies — quasars

\section{INTRODUCTION}

Active Galactic Nuclei (AGNs), such as Seyfert galaxies, quasars and blazars (BL Lac objects and flat spectrum radio quasars) have been known to show variations in their continuum emission since their discovery (Smith \& Hoffleit 1963; Sandage 1964, 1966; Kinman 1968) and this is now one of their defining characteristics. The majority of quasars are variable and such continuum flux variability is of the order of up to tens of percent in amplitude (Giveon et al. 1999; Sesar et al. 2007). This is commonly aperiodic in nature and has been observed to occur on timescales of days to years and in all energy bands from X-ray to radio wavelengths (Hook et al. 1994; Vanden Berk et al. 2004). Also, optical flux variations within a night with amplitudes of variability of the order of a few tenths of a magnitude (and referred to as intra-night optical variability; INOV) have been known in both radio-loud and radio-quiet quasars (Stalin et al. 2009, 2004). Though debated, these INOVs might be related to hot spots on the accretion disk (Mangalam \& Wiita 1993) and/or associated with relativistic jets (Marscher et al. 1992). This particular flux variability of quasars has also been used as an efficient tool in finding new quasars from photometric monitoring data (Koo et al. 1986; Ivezic et al. 2004; Rengstorf et al. 2004; Palanque-Delabrouille et al. 2011; Kim et al. 2011; Schmidt et al. 2010). In spite of large observational and theoretical efforts to study quasar variability, we still do not have a clear understanding of the physical mechanisms causing flux variations, both their long term and INOV cases. It is generally thought that the UV/optical radiation from quasars is from a geometrically thin, optically thick accretion disk powered by a supermassive black hole at the center. Several models are available in literature to explain the long term optical flux variability in quasars covering a wide range of physical mechanisms, namely instabilities in the accretion disk (Kawaguchi et al. 1998; Siemiginowska \& Elvis 1997), multiple supernova explosions (Terlevich et al. 1992; Aretxaga et al. 1997), gravitational microlensing (Hawkins 1993), star collisions (Courvoisier et al. 1996; Torricelli-Ciamponi et al. 2000), and thermal fluctuations driven by a stochastic process 
(Kelly et al. 2009) and a random walk (MacLeod et al. 2010). However, the observed optical-UV variability of quasars is not explained successfully by any of the above models and thus the origin of quasar variability is still unclear.

Often the flux variations in AGNs in different energy bands are associated with time delays between them. Such a time lag between flux variations in different energy bands may hint at a common process connecting spatially separated regions in an accretion disk where most of the corresponding wavelengths are emitted (Bachev 2009). In the optical region, delays of the order of days have also been observed with short wavelength variations leading long wavelength variations (Cackett et al. 2007; Wanders et al. 1997; Collier 2001). To explain these observed optical variations, Krolik et al. (1991) suggested the reprocessing model. According to this model, the central high energy X-ray emission is reprocessed to optical photons from the outer and therefore colder regions of the accretion disk. Thus, if the observed optical variations are driven by changes in the central X-ray continuum, there should be a time lag between the optical $V$ and $R$ band variations with the $V$ band variations leading the $R$ band variations. Alternatively, shorter wavelength radiation in an AGN can originate closer to the accretion disk as the accretion disk temperature varies with the radius of the disk as $T \propto R^{-3 / 4}$. Thus in the case of accretion disk fluctuations drifting inwards (Arévalo et al. 2008), the $V$ band variations can lag the $R$ band variations. Therefore, examination of the time lags between the continuum changes associated with different optical bands for a large sample of quasars can help to constrain the models in literature that describe the optical flux variations in quasars. Bachev (2009), using the $B$ and $R$ band observations of 42 Palomar-Green (PG) quasars (Schmidt \& Green 1983), found that the red band variations lag behind the blue band variations, consistent with reprocessing models. Similar results were also reported by Sergeev et al. (2005) and Liu et al. (2008).

Flux variations in quasars are also generally associated with a change in their spectra. Such spectral variations in the blazar class of AGNs have been investigated by several authors on different timescales (Stalin et al. 2006, 2009; Carini et al. 2011; Poon et al. 2009; Ghisellini et al. 1997; Romero et al. 2000; Raiteri et al. 2003; Villata et al. 2000). These changes in quasar colour with their brightness could help in understanding their central engine. While monitoring 42 PG quasars over a period of seven years, Giveon et al. (1999) found that a large fraction of quasars in their sample become bluer as they become brighter. There are two explanations available in the literature for this observationally known bluer when brighter trend. One explanation is that the spectral hardening with brightness is due to a variable component becoming brighter and then getting bluer (Giveon et al. 1999; Wilhite et al. 2005; Wamsteker et al. 1990). The other explanation is that spectral hardening with brightness can be due to the variable component with constant blue colour becoming brighter and dominant over the non-variable component that has red colour (Winkler et al. 1992; Winkler 1997; Choloniewski 1981). Recently, using quasars in SDSS stripe 82, Sun et al. (2014) found a time dependent colour variation. According to Sun et al. (2014), colour variation decreases with the timescale of flux variations, and is prominent on short timescales of around 10 days. This finding by Sun et al. (2014) rules out models that attribute the bluer when brighter trend to a combination of variable emission with blue and constant colour and a redder non-variable emission. However, there are also studies that claim to have found no spectral hardening with brightness in quasars. Over a period of three years, Winkler (1997) monitored 91 Seyfert 1 galaxies and found a linear flux-to-flux relation in them. Similar results are also available in literature (Sakata et al. 2010; Tomita et al. 2006). Such a linear relationship between fluxes in any two optical bands can indicate constant optical colour or constant spectral shape of the variable component in those sources. Thus, based on available observations, it is still not known conclusively if the continuum flux changes in quasars are also accompanied by a change in their broadband spectra.

The main motivation for this work is to characterise the long term optical variability properties of quasars and their colour variations. Section 2 describes the sample used in the study, Section 3 explains the time lag determination, Section 4 discusses the optical colour variability and the results and conclusions are given in the final section.

\section{THE SAMPLE}

The sample of quasars used in this study was taken from the Massive Compact Halo Objects (MACHO) database. The Magellanic clouds were monitored by the MACHO project using the $1.27 \mathrm{~m}$ telescope at the Mount Stromlo Observatory in red (5900-7800 $\AA$ ) and blue (4370$5900 \AA$ A) bands between 1992 July and 2000 January, with the main aim of detecting Galactic micro-lensing events associated with the Magellanic clouds. The MACHO database contains light curves for a $7.5 \mathrm{yr}$ period in the standard $V$ and $R$ bands with a varying sampling frequency between 2 to 10 days. From this MACHO database, using variability criteria and subsequent spectroscopic follow up, a total of 59 quasars was found to lie behind the Magellanic clouds by Geha et al. (2003). As the sample quasars are selected from the MACHO database via a variability criterion, this sample is therefore biased towards highly variable sources. These quasars have good quality light curves in $V$ and $R$ bands with reasonably good temporal sampling. They span the redshift range $0.2<z<$ 2.8 and their apparent $V$ band magnitudes are between 16.6 and $20.1 \mathrm{mag}$. These quasars have light curves extending over a $7.5 \mathrm{yr}$ period with data points ranging between 49 and 1353 epochs. Their $V$ and $R$ band light curves were taken from the MACHO site ${ }^{1}$. The details of these objects are given in Table 1 . Here, column 2 is the MACHO iden-

\footnotetext{
1 http://www.ucolick.org/ mgeha/MACHO
} 
Table 1 Information about the MACHO Sample of Quasars Used in This Work

\begin{tabular}{|c|c|c|c|c|c|c|c|c|c|c|c|c|}
\hline No. & MACHO ID & $\alpha_{(2000)}$ & $\delta_{(2000)}$ & $V$ & $V-R$ & $z$ & Npts & $A_{V}$ & $A_{R}$ & $J$ & $H$ & $K$ \\
\hline 1 & 42.860 .123 & 04:46:11.14 & $-72: 05: 09.80$ & 17.60 & 0.29 & 0.95 & 49 & - & - & 16.03 & 15.35 & 15.12 \\
\hline 2 & 17.2227 .488 & 04:53:56.55 & $-69: 40: 35.96$ & 18.88 & 0.32 & 0.28 & 418 & 0.26 & 0.21 & & & \\
\hline 3 & 48.2620 .2719 & 04:56:14.19 & $-67: 39: 10.81$ & 19.03 & 0.32 & 0.26 & 335 & 0.19 & 0.16 & & & \\
\hline 4 & 17.3197 .1182 & 05:00:17.56 & $-69: 32: 16.32$ & 18.88 & 0.33 & 0.90 & 175 & 0.29 & 0.23 & & & \\
\hline 5 & 53.3360 .344 & 05:00:54.00 & $-66: 44: 01.34$ & 19.22 & 0.24 & 1.86 & 236 & 0.07 & 0.06 & & & \\
\hline 6 & 25.3469 .117 & 05:01:46.68 & $-67: 32: 41.81$ & 18.07 & 0.26 & 0.38 & 327 & 0.10 & 0.08 & 16.64 & 15.44 & 14.54 \\
\hline 7 & 25.3712 .72 & 05:02:53.65 & $-67: 25: 46.44$ & 18.61 & 0.31 & 2.17 & 340 & 0.10 & 0.08 & & & \\
\hline 8 & 53.3725 .29 & 05:03:04.04 & $-66: 33: 46.62$ & 18.10 & 0.45 & 0.06 & 236 & - & - & 14.42 & 13.72 & 12.90 \\
\hline 9 & 53.3970 .140 & 05:04:36.01 & $-66: 24: 17.03$ & 18.50 & 0.27 & 2.04 & 98 & - & - & & & \\
\hline 10 & 1.4418 .1930 & 05:07:36.39 & $-68: 47: 52.94$ & 20.05 & 0.17 & 0.53 & 422 & 0.14 & 0.12 & & & \\
\hline 11 & 1.4537 .1642 & 05:08:31.89 & $-68: 55: 10.66$ & 19.75 & 0.22 & 0.61 & 434 & 0.22 & 0.17 & & & \\
\hline 12 & 52.4565 .356 & 05:08:30.64 & $-67: 02: 30.05$ & 19.16 & 0.22 & 2.29 & 243 & 0.12 & 0.10 & & & \\
\hline 13 & 9.4641 .568 & 05:08:45.95 & $-70: 05: 00.92$ & 19.20 & 0.30 & 1.18 & 894 & 0.19 & 0.16 & & & \\
\hline 14 & 5.4643 .149 & 05:09:15.49 & $-69: 54: 16.75$ & 17.95 & 0.33 & 0.17 & 883 & 0.17 & 0.14 & 15.96 & 15.40 & 14.54 \\
\hline 15 & 20.4678 .600 & 05:08:54.08 & $-67: 37: 35.57$ & 20.06 & 0.24 & 2.22 & 349 & 0.12 & 0.10 & & & \\
\hline 16 & 9.4882 .332 & $05: 10: 23.18$ & $-70: 07: 36.12$ & 18.83 & 0.33 & 0.32 & 902 & 0.14 & 0.12 & & & \\
\hline 17 & 5.4892 .1971 & $05: 10: 32.32$ & $-69: 27: 16.90$ & 18.45 & 0.33 & 1.58 & 886 & 0.19 & 0.16 & & & \\
\hline 18 & 22.4990 .462 & 05:11:40.77 & $-71: 00: 32.95$ & 19.82 & 0.40 & 1.56 & 488 & 0.17 & 0.14 & & & \\
\hline 19 & 9.5239 .505 & $05: 12: 59.56$ & $-70: 30: 24.76$ & 19.18 & 0.36 & 1.30 & 943 & 0.19 & 0.16 & & & \\
\hline 20 & 9.5484 .258 & 05:14:12.05 & $-70: 20: 25.64$ & 18.61 & 0.33 & 2.32 & 404 & 0.22 & 0.17 & & & \\
\hline 21 & 37.5584 .159 & 05:15:04.72 & $-71: 43: 38.62$ & 19.43 & 0.67 & 0.50 & 246 & - & - & & & \\
\hline 22 & 22.5595 .1333 & $05: 15: 22.94$ & $-70: 58: 06.77$ & 18.55 & 0.29 & 1.15 & 234 & 0.12 & 0.10 & & & \\
\hline 23 & 13.5717 .178 & 05:15:36.02 & $-70: 54: 01.65$ & 18.56 & 0.37 & 1.66 & 486 & 0.12 & 0.10 & & & \\
\hline 24 & 78.5855 .788 & $05: 16: 26.23$ & $-69: 48: 19.39$ & 18.61 & 0.22 & 0.63 & 440 & 0.22 & 0.17 & & & \\
\hline 25 & 2.5873 .82 & 05:16:28.78 & $-68: 37: 02.38$ & 17.44 & 0.44 & 0.46 & 948 & 0.22 & 0.17 & 15.43 & 14.79 & 14.08 \\
\hline 26 & 58.5903 .69 & $05: 16: 36.76$ & $-66: 34: 36.92$ & 18.20 & 0.26 & 2.24 & 225 & - & - & & & \\
\hline 27 & 13.5962 .237 & 05:17:17.03 & $-70: 44: 02.46$ & 19.33 & 0.46 & 0.17 & 852 & 0.17 & 0.14 & & & \\
\hline 28 & 58.6272 .729 & 05:18:51.97 & $-66: 09: 56.70$ & 19.85 & 0.37 & 1.53 & 126 & - & - & & & \\
\hline 29 & 59.6398 .185 & 05:19:28.02 & $-65: 49: 50.50$ & 19.33 & 0.37 & 1.64 & 270 & - & - & & & \\
\hline 30 & 6.6572 .268 & 05:20:56.93 & $-70: 24: 52.50$ & 18.33 & 0.24 & 1.81 & 956 & 0.19 & 0.16 & & & \\
\hline 31 & 63.6643 .393 & 05:20:56.45 & $-65: 39: 04.79$ & 19.65 & 0.41 & 0.47 & 234 & - & - & & & \\
\hline 32 & 13.6805 .324 & $05: 22: 47.23$ & $-71: 01: 31.08$ & 18.66 & 0.31 & 1.72 & 881 & 0.17 & 0.14 & & & \\
\hline 33 & 13.6808 .521 & $05: 22: 47.69$ & $-70: 47: 34.82$ & 19.02 & 0.33 & 1.64 & 386 & 0.22 & 0.17 & & & \\
\hline 34 & 6.7059 .207 & $05: 24: 02.31$ & $-70: 11: 08.95$ & 18.26 & 0.47 & 0.15 & 419 & 0.12 & 0.10 & 15.43 & 14.83 & 13.88 \\
\hline 35 & 63.7365 .151 & $05: 25: 14.29$ & $-65: 54: 45.93$ & 18.72 & 0.33 & 0.65 & 237 & - & - & & & \\
\hline 36 & 77.7551 .3853 & 05:27:16.19 & $-69: 39: 33.96$ & 19.75 & - & 0.85 & 1353 & 0.12 & 0.10 & & & \\
\hline 37 & 61.8072 .358 & 05:30:07.93 & $-67: 10: 27.20$ & 19.33 & 0.27 & 1.65 & 216 & - & - & & & \\
\hline 38 & 64.8088 .215 & 05:30:09.06 & $-66: 07: 01.05$ & 18.96 & 0.23 & 1.95 & 220 & - & - & & & \\
\hline 39 & 64.8092 .454 & 05:30:08.75 & $-65: 51: 24.27$ & 20.10 & 0.21 & 2.03 & 231 & - & - & & & \\
\hline 40 & 61.8199 .302 & 05:30:26.81 & $-66: 48: 55.31$ & 18.94 & 0.26 & 1.79 & 348 & - & - & & & \\
\hline 41 & 14.8249 .74 & 05:31:31.60 & $-71: 29: 47.78$ & 19.36 & 0.28 & 0.22 & 427 & 0.29 & 0.23 & & & \\
\hline 42 & 82.8403 .551 & 05:31:59.66 & $-69: 19: 51.12$ & 19.40 & 0.33 & 0.15 & 794 & 0.12 & 0.10 & 16.22 & 15.84 & 15.10 \\
\hline 43 & 11.8988 .1350 & 05:36:00.50 & $-70: 41: 28.86$ & 19.52 & 0.3 & 0.33 & 937 & 0.26 & 0.21 & & & \\
\hline 44 & 68.10968 .454 & $05: 47: 45.13$ & $-67: 45: 5.745$ & 20.45 & 0.56 & 0.39 & 212 & - & - & & & \\
\hline 45 & 68.10972 .36 & $05: 47: 50.18$ & $-67: 28: 02.44$ & 16.63 & 0.28 & 1.01 & 234 & - & - & 15.04 & 14.69 & 14.01 \\
\hline 46 & 30.11301 .499 & 05:49:41.63 & $-69: 44: 15.86$ & 19.41 & 0.37 & 0.46 & 263 & 0.29 & 0.23 & 16.26 & 15.64 & 14.31 \\
\hline 47 & 28.11400 .609 & 05:50:31.22 & $-71: 09: 58.47$ & 20.08 & 0.31 & 0.44 & 307 & 0.19 & 0.16 & & & \\
\hline 48 & 70.11469 .82 & 05:50:33.31 & $-66: 36: 52.96$ & 18.19 & 0.66 & 0.08 & 238 & - & - & 15.06 & 14.30 & 13.70 \\
\hline 49 & 69.12549 .21 & $05: 57: 22.41$ & $-67: 13: 22.16$ & 17.41 & 0.43 & 0.14 & 235 & - & - & 14.88 & 13.89 & 12.66 \\
\hline 50 & 75.13376 .66 & $06: 02: 34.25$ & $-68: 30: 41.51$ & 18.63 & 0.26 & 1.07 & 209 & 0.10 & 0.08 & & & \\
\hline 51 & 208.15799 .1085 & $00: 47: 15.76$ & $-72: 41: 12.24$ & 19.52 & 0.28 & 2.77 & 800 & 0.07 & 0.06 & & & \\
\hline 52 & 208.15920 .619 & $00: 49: 34.43$ & $-72: 13: 08.99$ & 19.28 & 0.19 & 0.91 & 711 & 0.07 & 0.06 & & & \\
\hline 53 & 208.16034 .100 & 00:51:16.89 & $-72: 16: 51.06$ & 18.03 & 0.26 & 0.49 & 238 & 0.12 & 0.10 & 16.71 & 16.10 & 15.21 \\
\hline 54 & 207.16310 .1050 & 00:55:59.61 & $-72: 52: 45.15$ & 19.17 & 0.32 & 1.47 & 787 & 0.22 & 0.17 & & & \\
\hline 55 & 207.16316 .446 & $00: 55: 34.70$ & $-72: 28: 34.23$ & 18.64 & 0.19 & 0.56 & 699 & 0.12 & 0.10 & & & \\
\hline 56 & 206.16653 .987 & 01:01:27.81 & $-72: 46: 14.37$ & 19.51 & 0.25 & 1.05 & 540 & 0.07 & 0.06 & & & \\
\hline 57 & 211.16703 .311 & 01:02:14.36 & $-73: 16: 26.80$ & 18.92 & 0.35 & 2.18 & 713 & 0.12 & 0.10 & & & \\
\hline 58 & 211.16765 .212 & 01:02:34.73 & $-72: 54: 22.20$ & 18.15 & 0.29 & 2.13 & 189 & 0.07 & 0.06 & & & \\
\hline 59 & 206.17052 .388 & 01:07:21.71 & $-72: 48: 45.76$ & 18.85 & 0.23 & 2.15 & 307 & 0.10 & 0.08 & & & \\
\hline
\end{tabular}


tification number, columns 3 and 4 are the right accession and declination respectively in the J2000 epoch, column 5 is the $V$ band magnitude, column 6 is the $V-R$ colour in magnitude, column 7 is the redshift, column 8 is the number of data points in the light curve, columns 9 and 10 are the extinction coefficients in $V$ and $R$ bands respectively and the last three columns give the infrared $J, H$ and $K$ band magnitudes where available.

\section{ANALYSIS}

All the collected light curves were corrected for Galactic and Magellanic cloud extinction. The Magellanic cloud extinction values were taken from the German Astrophysical Virtual Observatory (http://dc.zah.uni-heidelberg.de/mcx) which uses the new reddening maps published by Haschke et al. (2011) based on data from the third phase of the Optical Gravitational Lensing Experiment (OGLE III) and the transformation relations from Schlegel et al. (1998). The Galactic extinction values were taken from $\mathrm{NED}^{2}$. We rejected those epochs having either $V$ band or $R$ band measurements from the light curves, so that these light curves contain data points having both simultaneous or nearly simultaneous $R$ and $V$ band measurements. The final light curves used in this study thus have 49 to 1353 data points spanning about $7.5 \mathrm{yr}$.

\subsection{Flux Variability}

To characterise the flux variation of a source, we have used the normalised excess variance given by Vaughan et al. (2003). This is defined as

$$
F_{\mathrm{var}}=\sqrt{\frac{S^{2}-\overline{\sigma_{\mathrm{err}}^{2}}}{\bar{x}^{2}}},
$$

where $\overline{\sigma_{\text {err }}^{2}}$ is the mean square error given as

$$
\overline{\sigma_{\mathrm{err}}^{2}}=\frac{1}{N} \sum_{i=1}^{N} \sigma_{\mathrm{err}, i}^{2}
$$

and $S^{2}$ is the sample variance defined as

$$
S^{2}=\frac{1}{N-1} \sum_{i}\left(x_{i}-\bar{x}\right)^{2} .
$$

The error in $F_{\text {var }}$ is calculated again using Vaughan et al. (2003) and is defined as

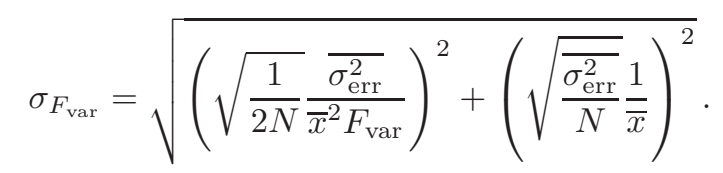

In Figure 1 is shown the plot of $F_{\text {var }}$ in $V$ band versus $R$ band for all the sources. It is evident from this figure that the majority of sources show more variations in the shorter

\footnotetext{
2 http://nedwww.ipac.caltech.edu/
}

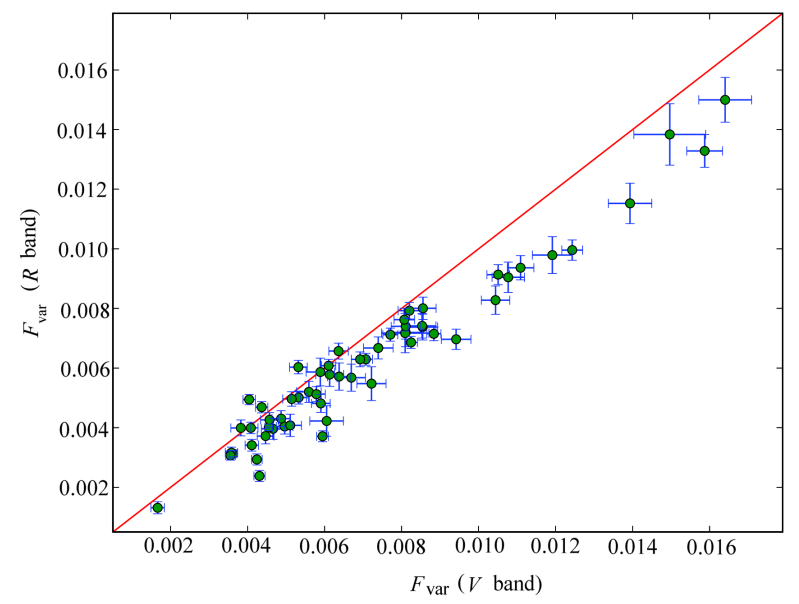

Fig. 1 Plot of the normalised excess variance in $V$ and $R$ bands for the quasars.

wavelength ( $V$ band) compared to the longer wavelength ( $R$ band), however a minority of sources show more variation in $R$ band relative to $V$ band. We note that Magdis \& Papadakis (2006), using the same MACHO database, found all quasars show larger variations in the $V$ band relative to the $R$ band, independent of their luminosity or redshift. This is in general agreement with our findings reported here.

\subsection{Color Variation}

Most of the results related to colour variations of quasars available in the literature are based on fitting the colours of quasars versus their magnitudes. These model fits do not take into account the inherent correlation between errors in the magnitudes and errors in colours estimated using those magnitudes which can lead to spurious results as pointed out by Schmidt et al. (2012). Therefore, in order to estimate the true colour variability we have adopted the Bayesian linear fitting procedure using the Markov Chain Monte Carlo (MCMC) method as described in Hogg et al. (2010) and Schmidt et al. (2012). We also compare the results with those obtained from three other different approaches, namely (a) performing a simple ordinary least squares (OLS) fit on data points in the colour versus magnitude plane, (b) performing a weighted linear least squares fit that takes into account the errors in both colours and magnitudes using the FITEXY routine of Press et al. (1992) and (c) the bivariate correlated errors and intrinsic scatter regression (BCES) method of Akritas \& Bershady (1996).

\subsubsection{MCMC method}

The MCMC method used for fitting the data takes into account the errors in both $V$ and $R$ bands and also prunes the outlier data points as detailed in Hogg et al. (2010). The details of how to use the MCMC method to find true colour variability in quasars are given in Schmidt et al. (2012). 


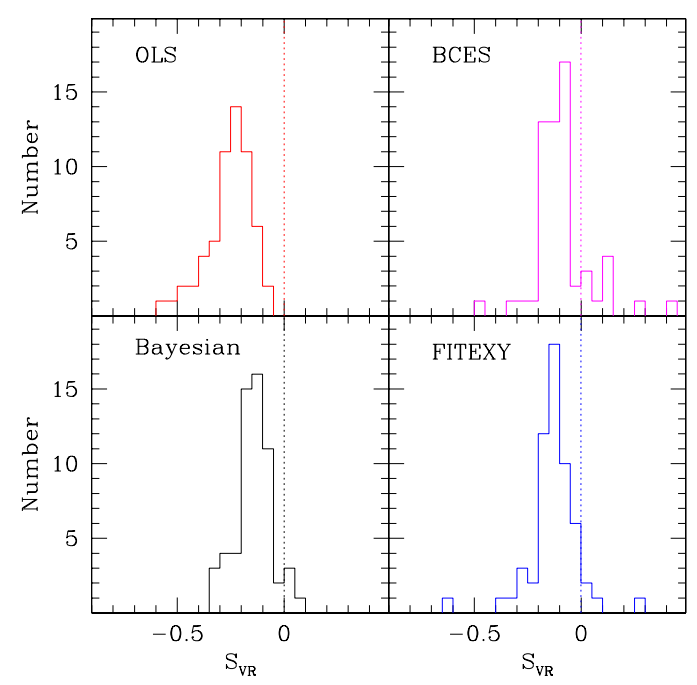

Fig. 2 Distribution of the colour variation of quasars. Here, the top left panel is from the OLS algorithm, the top right panel shows the results of the BCES method, and the bottom left and right panels are from the MCMC and FITEXY algorithms respectively.

Here, we briefly describe the fitting procedure. A linear fit of the general form for the mean subtracted data is

$$
R-\langle R\rangle=S_{V R}(V-\langle V\rangle)+b,
$$

where $V$ and $R$ are the observed $V$ and $R$ band magnitudes respectively and $\langle V\rangle$ and $\langle R\rangle$ are the mean values of $V$ and $R$ band magnitudes respectively. This fit can be interpreted as a fit in the $V$ - $R$ colour versus $V$-magnitude space of the following equation obtained from simple algebraic operations applied to Equation (5)

$$
V-R=-\left(S_{V R}-1\right)(V-\langle V\rangle)+B,
$$

where $B=-b+(\langle V\rangle-\langle R\rangle)$. According to the above equation, if $S_{V R}<1$, the quasar becomes bluer when brighter, if $S_{V R}=1$, there is no colour variability and if $S_{V R}>1$ the quasar becomes redder when brighter. A large space of the parameters $\left(S_{V R}\right.$ and $\left.b\right)$ are sampled using an MCMC chain. The best fit parameters are obtained using the peak of the resulting distributions.

The results of our colour analysis are displayed in Figure 2. From the figure it is clear that using the OLS algorithm, all quasars show a bluer when brighter trend. However, when using the other three approaches, namely FITEXY, BCES and MCMC, although the majority of quasars show a bluer when brighter trend, a minority of sources show a redder when brighter behaviour. Our analysis of these 59 quasars shows that, when studying the colour variations in quasars, one needs to properly take into account the correlation between the errors in colours and magnitudes as pointed out by Schmidt et al. (2012).

\section{TIME LAG BETWEEN $V$ AND $R$ BANDS}

The time lag between $V$ and $R$ bands of all the 59 objects was studied using the z-transformed discrete correla- tion function (zDCF, Alexander 2014, 1997). This method is well suited to estimate the cross-correlation between sparse, unevenly sampled light curves. Unlike the commonly used interpolation method, zDCF does not assume that the light curves are smooth, and it also gives an estimate of the errors in the cross correlation function (Alexander 1997; Liu et al. 2008). We found no lag between $V$ and $R$ band flux variations for the quasars in our sample. Similar results have been obtained by Magdis \& Papadakis (2006). They too found no lag between $V$ and $R$ band flux variations in most of the MACHO sources. However, in some sources they noticed the $V$ band variations leading the $R$ band variations.

\section{CONCLUSIONS}

We carried out a systematic analysis of the long term flux and colour variations of quasars using a sample of 59 sources selected from the MACHO database. The main findings of this study are as follows:

(1) All the quasars showed long term variations during the $7.5 \mathrm{yr}$ duration of observations.

(2) A large fraction of the quasars showed large amplitude variations in the shorter wavelength $V$ band compared to the longer wavelength $R$ band.

(3) No time lag is noticed between the flux variations in the $V$ and $R$ bands.

(4) Using a Bayesian linear fit with an MCMC algorithm that takes into account the correlation between the errors in colour and magnitude, it is found that most of the sources show a bluer when brighter trend.

(5) Similar results are also obtained by using the FITEXY and BCES algorithms. Alternatively, if an OLS fitting is done which is normally followed in the study of colour variations in quasars, we found that all the quasars show a bluer when brighter trend. This is in contrast to the results obtained using the other three algorithms. Therefore, it is clear that when studying the spectral variations in quasars using colour magnitude diagrams, the correlation between the errors in colours and magnitudes needs to be taken into account.

Quasars in the MACHO database have also been studied for spectral variations by Vagnetti \& Trevese (2006) and flux variations by Magdis \& Papadakis (2006). Some of the results presented in this work are in general agreement with those reported by Magdis \& Papadakis (2006).

Acknowledgements N. Sukanya thanks the University Grants Commission (UGC) for the award of a BSR Fellowship for a period of one year to carry out this work. This research has made use of the NASA Extragalactic Database (NED). Arnab Dhani thanks the Indian Institute of Astrophysics for a short term internship at IIA during which some work related to the idea of this paper was carried out. S. Jeyakumar acknowledges the hospitality provided to him for his visit to IIA, when part of the work was done. 


\section{References}

Akritas, M. G., \& Bershady, M. A. 1996, ApJ, 470, 706

Alexander, T. 1997, in Astrophysics and Space Science Library,

218, Astronomical Time Series, eds. D. Maoz, A. Sternberg,

\& E. M. Leibowitz, 163

Alexander, T. 2014, ZDCF: Z-Transformed Discrete Correlation

Function, Astrophysics Source Code Library, ascl:1404.002

Aretxaga, I., Cid Fernandes, R., \& Terlevich, R. J. 1997, MNRAS, 286, 271

Arévalo, P., Uttley, P., Kaspi, S., et al. 2008, MNRAS, 389, 1479

Bachev, R. S. 2009, A\&A, 493, 907

Cackett, E. M., Horne, K., \& Winkler, H. 2007, MNRAS, 380, 669

Carini, M. T., Walters, R., \& Hopper, L. 2011, AJ, 141, 49

Choloniewski, J. 1981, Acta Astronomica, 31, 293

Collier, S. 2001, MNRAS, 325, 1527

Courvoisier, T. J.-L., Paltani, S., \& Walter, R. 1996, A\&A, 308, L17

Geha, M., Alcock, C., Allsman, R. A., et al. 2003, AJ, 125, 1

Ghisellini, G., Villata, M., Raiteri, C. M., et al. 1997, A\&A, 327, 61

Giveon, U., Maoz, D., Kaspi, S., Netzer, H., \& Smith, P. S. 1999, MNRAS, 306, 637

Haschke, R., Grebel, E. K., \& Duffau, S. 2011, AJ, 141, 158

Hawkins, M. R. S. 1993, Nature, 366, 242

Hogg, D. W., Bovy, J., \& Lang, D. 2010, arXiv:1008.4686

Hook, I. M., McMahon, R. G., Boyle, B. J., \& Irwin, M. J. 1994, MNRAS, 268, 305

Ivezic, Ž., Lupton, R. H., Juric, M., et al. 2004, in IAU Symposium, 222, The Interplay Among Black Holes, Stars and ISM in Galactic Nuclei, eds. T. Storchi-Bergmann, L. C. Ho, \& H. R. Schmitt, 525

Kawaguchi, T., Mineshige, S., Umemura, M., \& Turner, E. L. 1998, in IAU Symposium, 188, The Hot Universe, eds. K. Koyama, S. Kitamoto, \& M. Itoh, 451

Kelly, B. C., Bechtold, J., \& Siemiginowska, A. 2009, ApJ, 698, 895

Kim, D.-W., Protopapas, P., Byun, Y.-I., et al. 2011, ApJ, 735, 68

Kinman, T. D. 1968, Science, 162, 1081

Koo, D. C., Kron, R. G., \& Cudworth, K. M. 1986, PASP, 98, 285

Krolik, J. H., Horne, K., Kallman, T. R., et al. 1991, ApJ, 371, 541

Liu, H. T., Bai, J. M., Zhao, X. H., \& Ma, L. 2008, ApJ, 677, 884

MacLeod, C. L., Ivezić, Ž., Kochanek, C. S., et al. 2010, ApJ, 721,1014

Magdis, G., \& Papadakis, I. E. 2006, in Astronomical Society of the Pacific Conference Series, 360, eds. C. M. Gaskell, I. M.

McHardy, B. M. Peterson, \& S. G. Sergeev, 37

Mangalam, A. V., \& Wiita, P. J. 1993, ApJ, 406, 420

Marscher, A. P., Gear, W. K., \& Travis, J. P. 1992, in Variability of Blazars, eds. E. Valtaoja, \& M. Valtonen, 85

Palanque-Delabrouille, N., Yeche, C., Myers, A. D., et al. 2011, A\&A, 530, A122

Poon, H., Fan, J. H., \& Fu, J. N. 2009, ApJS, 185, 511
Press, W. H., Teukolsky, S. A., Vetterling, W. T., \& Flannery, B. P. 1992, Numerical Recipes in FORTRAN. The Art of Scientific Computing (Cambridge: Cambridge Univ. Press)

Raiteri, C. M., Villata, M., Tosti, G., et al. 2003, A\&A, 402, 151

Rengstorf, A. W., Mufson, S. L., Andrews, P., et al. 2004, ApJ, 617, 184

Romero, G. E., Cellone, S. A., \& Combi, J. A. 2000, AJ, 120, 1192

Sakata, Y., Minezaki, T., Yoshii, Y., et al. 2010, ApJ, 711, 461

Sandage, A. 1964, ApJ, 139, 416

Sandage, A. 1966, ApJ, 144, 1234

Schlegel, D. J., Finkbeiner, D. P., \& Davis, M. 1998, ApJ, 500, 525

Schmidt, K. B., Rix, H.-W., Jester, S., et al. 2010, in IAU Symposium, 267, eds. B. M. Peterson, R. S. Somerville, \& T. Storchi-Bergmann, 265

Schmidt, K. B., Rix, H.-W., Shields, J. C., et al. 2012, ApJ, 744, 147

Schmidt, M., \& Green, R. F. 1983, ApJ, 269, 352

Sergeev, S. G., Doroshenko, V. T., Golubinskiy, Y. V., Merkulova, N. I., \& Sergeeva, E. A. 2005, ApJ, 622, 129

Sesar, B., Ivezić, Ž., Lupton, R. H., et al. 2007, AJ, 134, 2236

Siemiginowska, A., \& Elvis, M. 1997, ApJ, 482, L9

Smith, H. J., \& Hoffleit, D. 1963, AJ, 68, 292

Stalin, C. S., Gopal-Krishna, Sagar, R., \& Wiita, P. J. 2004, MNRAS, 350, 175

Stalin, C. S., Gopal-Krishna, Sagar, R., et al. 2006, MNRAS, 366, 1337

Stalin, C. S., Kawabata, K. S., Uemura, M., et al. 2009, MNRAS, 399, 1357

Sun, Y.-H., Wang, J.-X., Chen, X.-Y., \& Zheng, Z.-Y. 2014, ApJ, 792, 54

Terlevich, R., Tenorio-Tagle, G., Franco, J., \& Melnick, J. 1992, MNRAS, 255, 713

Tomita, H., Yoshii, Y., Kobayashi, Y., et al. 2006, ApJ, 652, L13

Torricelli-Ciamponi, G., Foellmi, C., Courvoisier, T. J.-L., \& Paltani, S. 2000, A\&A, 358, 57

Vagnetti, F., \& Trevese, D. 2006, in Astronomical Society of the Pacific Conference Series, 360, eds. C. M. Gaskell, I. M. McHardy, B. M. Peterson, \& S. G. Sergeev, 33

Vanden Berk, D. E., Wilhite, B. C., Kron, R. G., et al. 2004, ApJ, 601,692

Vaughan, S., Edelson, R., Warwick, R. S., \& Uttley, P. 2003, MNRAS, 345, 1271

Villata, M., Raiteri, C. M., Popescu, M. D., et al. 2000, A\&AS, 144,481

Wamsteker, W., Rodriguez-Pascual, P., Wills, B. J., et al. 1990, ApJ, 354, 446

Wanders, I., Peterson, B. M., Alloin, D., et al. 1997, ApJS, 113, 69

Wilhite, B. C., Vanden Berk, D. E., Kron, R. G., et al. 2005, ApJ, 633,638

Winkler, H. 1997, MNRAS, 292, 273

Winkler, H., Glass, I. S., van Wyk, F., et al. 1992, MNRAS, 257, 659 